\title{
LEMME DE FATOU POUR L'INTÉGRALE DE PETTIS
}

\author{
Allal Amrani
}

\begin{abstract}
The purpose of this paper is to present Fatou type results for a sequence of Pettis integrable functions and multifunctions. We prove the non vacuity of the weak upper limit of a sequence of Pettis integrable functions taking their values in a locally convex space and we deduce a Fatou's lemma for a sequence of convex weak compact valued Pettis integrable multifunctions. We prove as well a Lebesgue theorem for a sequence of Pettis integrable multifunctions with values in the space of convex compact sets of a separable Banach space.
\end{abstract}

\section{Introduction.}

Le lemme de Fatou formulé en termes de fonctions univoques est connu pour son utilité dans les problèmes variationnels, d'économie mathématique et de contrôle optimal. Les premiers travaux dans cette direction remontent à Aumann $[\mathbf{A u}]$ et Artstein $[\mathbf{A r}]$. Il y a eu par la suite un nombre considérable de travaux généralisant dans diverses directions les résultats précédant, citons à titre d'exemple Yannelis $[\mathbf{Y}]$, Hess $[\mathbf{H}]$, Castaing-Clauzure $[\mathbf{C C}]$ et plus particulièrement Balder $[\mathbf{B 1}]$. Pour une étude englobant et généralisant la plupart des résultats précédants, on pourra consulter l'article de Balder et Hess $[\mathbf{B H}]$.

Notre objectif est d'établir des résultats similaires aux précédents pour les fonctions et multifonctions Pettis-intégrables. Les démonstrations s'appuient sur des développements récents de la théorie de l'intégrale de Pettis dûs à Musial $[\mathbf{M}]$, Geitz $[\mathbf{G e}]$ et Huff $[\mathbf{H u}]$. Dans le paragraphe 2, on commence par démontrer la non vacuité de la limite supérieure faible d'une suite de fonctions Pettis-intégrables puis l'on déduit le lemme

Keywords. Multifunction, Pettis, Komlos, Lebesgue, Fatou.

1991 Mathematics subject classifications: 28B20, 26E25. 
de Fatou pour des multifonctions Pettis-intégrables à valeurs dans les parties convexes faiblement compactes d'un espace localement convexe séparé. Le paragraphe 3 est consacré aut théorème de Lebesgue pour des multifonctions à valeurs convexe compactes d'un espaces de Banach séparable.

\section{Notations et Définitions.}

Soit $(E, \tau)$ un espace localement convexe séparé, quasi-complet et $E^{\prime}$ son dual topologique. On désigne par $\operatorname{ck}(E)(\operatorname{resp} \cdot \operatorname{cwk}(E))$, l'ensemble des parties convexes compactes (resp. faiblement compactes) de $E$. On note $\bar{A}$ l'adhérence de $A, \operatorname{co}(A)$ l'enveloppe convexe de $A, \overline{\mathrm{co}}(A)$ l'enveloppe convexe fermée de $A$. Si $\left(D_{n}\right)$ est une suite de parties de $E$, on notera $\operatorname{Ls}\left(D_{n}\right)$, l'ensemble $\cap_{k \geq 1} \overline{\left(\cup_{n \geq k} D_{n}\right)}$. On obtient la limite supérieure faible, notée $\mathrm{w}-\operatorname{Ls}\left(D_{n}\right)$, en prenant les adhérences pour la topologie faible. Pour la topologie faible, il y a aussi la limite supérieure séquentielle faible, notée seq-w-Ls $\left(D_{n}\right)$, qui est l'ensemble des limites des suites faiblement convergentes $\left(x_{k}\right)_{k \in \mathbb{N}}$ oú $\left(x_{k}\right) \in D_{n_{k}},\left(n_{k}\right)_{k \in \mathbb{N}}$ étant une suite strictement croissante d'entiers.

Soit $(\Omega, \mathcal{A}, \mu)$ un espace mesuré avec $\mu \sigma$-finie et $\mathcal{A} \mu$-complete. Une application $f: \Omega \rightarrow E$ est scalairement mesurable (resp. scalairement intégrable), si pour tout $x^{\prime} \in E^{\prime},\left\langle x^{\prime}, f\right\rangle$ est mesurable (resp. intégrable). Une application scalairement intégrable $f$ est Pettis-intégrable, si il existe une fonction d'ensemble $\nu_{f}: \mathcal{A} \rightarrow E$ telle que pour tout $x^{\prime} \in E^{\prime}$ et pour tout $A \in \mathcal{A}$, on ait:

$$
\int_{A}\left\langle x^{\prime}, f\right\rangle d \mu=\left\langle x^{\prime}, \nu_{f}(A)\right\rangle
$$

$\nu_{f}(A)$ est l'intégrale de Pettis de $f$ sur $A$ et sera notée dans la suite $\int_{A} f d \mu$. Une multifunction $\Gamma: \Omega \rightarrow \operatorname{cwk}(E)$ est scalairement mesurable (resp. intégrable) si pour tout $x^{\prime} \in E^{\prime}, \delta^{*}\left(x^{\prime}, \Gamma(\cdot)\right)$ est mesurable (resp. intégrable). Une multifonction $\Gamma$ scalairement intégrable est faiblement Pettis-intégrable (resp. fortement Pettis-intégrable) si il existe une fonction d'ensemble $K: \mathcal{A} \rightarrow \operatorname{cwk}(E)$ (resp. $\operatorname{ck}(E)$ ) telle que pour tout $x^{\prime} \in E^{\prime}$ et pour tout $A \in \mathcal{A}$, on ait:

$$
\int_{A} \delta^{*}\left(x^{\prime}, \Gamma\right) d \mu=\delta^{*}\left(x^{\prime}, K(A)\right) .
$$

On note $S_{\Gamma}$ l'ensemble des sections scalairement intégrable de $\Gamma$.

\section{Lemme de Fatou pour l'intégrale de Pettis.}

Le lemme technique suivant servira dans la démonstration du résultat principal de ce paragraphe. 
Lemme 3.1. Soient $E$ un espace topologique séparé, $K$ un compact de E. Soit $\left(x_{n}\right)_{n \in \mathbb{N}}$ une suite dans $K, x \in E$, et $x^{\prime} \in E^{\prime}$, alors:

$$
\left\langle x^{\prime}, x\right\rangle \in \overline{\mathrm{co}} \operatorname{Ls}\left(\left\langle x^{\prime}, x_{n}\right\rangle\right) \Rightarrow\left\langle x^{\prime}, x\right\rangle \leq \delta^{*}\left(x^{\prime} ; \overline{\mathrm{CO}} \operatorname{Ls}\left(x_{n}\right)\right) .
$$

Démonstration: Comme la suite $\left(x_{n}\right)_{n \in \mathbb{N}}$ demeure dans le compact $K$, la suite $\left(\left\langle x^{\prime}, x_{n}\right\rangle\right)_{n \in \mathbb{N}}$ est bornée dans $\mathbb{R}$, donc $\operatorname{Ls}\left(\left\langle x^{\prime}, x_{n}\right\rangle\right)$ est compact. Il s'ensuit que

$$
\overline{\operatorname{co}} \operatorname{Ls}\left(\left\langle x^{\prime}, x_{n}\right\rangle\right)=\operatorname{coLs}\left(\left\langle x^{\prime}, x_{n}\right\rangle\right) .
$$

Donc $\left\langle x^{\prime}, x\right\rangle=\sum_{i=1}^{k} \lambda_{i} z_{i}$ avec $\sum_{i=1}^{k} \lambda_{i}=1$ et $z_{i} \in \operatorname{Ls}\left(\left\langle x^{\prime}, x_{n}\right\rangle\right)$. Comme la fonction d'appui est positivement homogène, le lemme sera démontré si l'on montre que pour tout $z \in \operatorname{Ls}\left(\left\langle x^{\prime}, x_{n}\right\rangle\right)$, on a $z \leq \delta^{*}\left(x^{\prime} ; \overline{\operatorname{co}} \operatorname{Ls}\left(x_{n}\right)\right)$. Si $z \in \operatorname{Ls}\left(\left\langle x^{\prime}, x_{n}\right\rangle\right)$, alors $z=\lim _{k \rightarrow+\infty}\left\langle x^{\prime}, x_{n_{k}}\right\rangle$. Par compacité de $K$, la suite $\left(x_{n_{k}}\right)_{k \in \mathbb{N}}$ admet une valeur d'adhèrence $\bar{x} \in K$, on a alors

$$
z=\left\langle x^{\prime}, \bar{x}\right\rangle \leq \delta^{*}\left(x^{\prime} ; \overline{\operatorname{co}} \operatorname{Ls}\left(x_{n}\right)\right) .
$$

Le théorème suivant est la clé des résultats de ce premier paragraphe. Il a été établi par Musial $[\mathbf{M}]$ et Geitz $[\mathbf{G e}]$. Nous donnons une démonstration plus courte basée sur un résultat profond dû à Grothendieck et sur le théorème de Komlos [Ko].

Théorème 3.2. Soient $\left(f_{n}\right)_{n \in \mathbb{N}}$ une suite de fonctions Pettis-intégrables et $f: \Omega \rightarrow E$ une application scalairement intégrable telles que:

(1) Pour toute partie équicontinue $B \subset E^{\prime}$, l'ensemble $\left\{\left\langle x^{\prime}, f\right\rangle: x^{\prime} \in\right.$ $B\}$ est relativement $\sigma\left(L^{1}, L^{\infty}\right)$ compact.

(2) Pour tout $x^{\prime} \in E^{\prime},\left\langle x^{\prime}, f_{n}\right\rangle$ converge vers $\left\langle x^{\prime}, f\right\rangle$ pour la topologie $\sigma\left(L^{1}, L^{\infty}\right)$.

Alors $f$ est Pettis-intégrable.

Démonstration: Grâce a (2), pour tout $x^{\prime} \in E^{\prime}$ et pour tout $A \in \mathcal{A}$, on a:

$$
\lim _{n \rightarrow \infty}\left\langle x^{\prime}, \int_{A} f_{n} d \mu\right\rangle=\lim _{n \rightarrow \infty} \int_{A}\left\langle x^{\prime}, f_{n}\right\rangle d \mu=\int_{A}\left\langle x^{\prime}, f\right\rangle d \mu .
$$

Par conséquent, il suffit de montrer que pour tout $A \in \mathcal{A}$, la suite $\left(\int_{A} f_{n} d \mu\right)_{n \in \mathbb{N}}$ est relativement faiblement compacte dans $E$. D'après le théorème d'Eberlein-Smulyan-Grothendieck ([G1, Corollaire 1 of Théorème 7]) (voir aussi [K, Theorem p. 326]) il est équivalent de montrer: 
pour toute partie équicontinue $B \subset E^{\prime}$, pour toute suite $\left(x_{k}^{\prime}\right)_{k \in \mathbb{N}} \subset B$ et pour toute sous-suite $\left(f_{n_{m}}\right)_{m \in \mathbb{N}}$ de $\left(f_{n}\right)_{n \in \mathbb{N}}$, on a:

$$
\alpha:=\lim _{k \rightarrow \infty} \lim _{m \rightarrow \infty}\left\langle x_{k}^{\prime}, \int_{A} f_{n_{m}} d \mu\right\rangle=\beta=: \lim _{m \rightarrow \infty} \lim _{k \rightarrow \infty}\left\langle x_{k}^{\prime}, \int_{A} f_{n_{m}} d \mu\right\rangle
$$

si ces ces limites existent. Du fait de (2), on a:

$$
\lim _{m \rightarrow \infty}\left\langle x_{k}^{\prime}, \int_{A} f_{n_{m}} d \mu\right\rangle=\lim _{m \rightarrow \infty} \int_{A}\left\langle x_{k}^{\prime}, f_{n_{m}}\right\rangle d \mu=\int_{A}\left\langle x_{k}^{\prime}, f\right\rangle d \mu .
$$

D'après le théorème de Komlos $[\mathbf{K o}]$, appliqué à la suite $\left(\left\langle x_{k}^{\prime}, f\right\rangle\right)_{k \in \mathbb{N}}$ il existe une suite $\left(y_{n}^{\prime}\right)_{n \in \mathbb{N}^{*}}$ avec $y_{n}^{\prime}=\frac{1}{n} \sum_{i=1}^{n} x_{k_{i}}^{\prime}$ et une fonction réelle intégrable $h$ telle que $\left\langle y_{n}^{\prime}, f\right\rangle$ converge vers $h$ presque partout. Du fait (3.1.2) et (1) on a

$$
\alpha=\lim _{k \rightarrow \infty} \int_{A}\left\langle x_{k}^{\prime}, f\right\rangle d \mu=\lim _{n \rightarrow \infty} \int_{A}\left\langle y_{n}^{\prime}, f\right\rangle d \mu=\int_{A} h d \mu .
$$

Soit $y_{0}^{\prime}$ une valeur d'adhérance faible $*$ de $\left(y_{n}^{\prime}\right)_{n \in \mathbb{N}^{*}}$, alors pour tout $m \in \mathbb{N}$, on a

$$
\begin{aligned}
\lim _{k \rightarrow \infty}\left\langle x_{k}^{\prime}, \int_{A} f_{n_{m}} d \mu\right\rangle & =\lim _{n \rightarrow \infty}\left\langle y_{n}^{\prime}, \int_{A} f_{n_{m}} d \mu\right\rangle \\
& =\left\langle y_{0}^{\prime}, \int_{A} f_{n_{m}} d \mu\right\rangle \\
& =\int_{A}\left\langle y_{0}^{\prime}, f_{n_{m}}\right\rangle d \mu .
\end{aligned}
$$

En prenant la limite quand $m \rightarrow \infty$ dans la dernière intégrale de (3.1.4) et en utilisant (2), on obtient

$$
\beta=\lim _{m \rightarrow \infty} \int_{A}\left\langle y_{0}^{\prime}, f_{n_{m}}\right\rangle d \mu=\int_{A}\left\langle y_{o}^{\prime}, f\right\rangle d \mu .
$$

Comme $\left\langle y_{n}^{\prime}, f\right\rangle$ converge vers $h$ presque partout et que $y_{0}^{\prime}$ est une valeur d'adhérance faible $*$ de $\left(y_{n}^{\prime}\right)_{n \in \mathbb{N}^{*}}, h=\left\langle y_{0}^{\prime}, f\right\rangle$ presque partout. Du fait de (3.1.1) et en utilisant (3.1.3), (3.1.4), (3.1.5) on obtient $\alpha=\beta$.

Dans la proposition qui suit on suppose que $(\Omega, \mathcal{A}, \mu)$ est sans atomes. 
Proposition 3.3. Soit $X$ une multiapplication à valeurs dans les parties fermées non vides de $E$. On suppose que $X$ admet au moins une section Pettis-intégrable; alors $\overline{\int_{\Omega} X(\omega) \mu(d \omega)}$ est convexe.

Démonstration: Il suffit de démontrer que pour toute suite finie $\left\{x_{1}^{\prime}, \ldots, x_{m}^{\prime}\right\}$ dans $E^{\prime}$, l'ensemble $A=\left\{\left(\left\langle x_{i}^{\prime}, x\right\rangle\right)_{i=1}^{m}, x \in \int_{\Omega} X(\omega) \mu(d \omega)\right\}$ est convexe dans $\mathbb{R}^{m}$. Si $y \in A$, alors $y=\left(\left\langle x_{i}^{\prime}, \int_{\Omega} f(\omega) \mu(d \omega)\right\rangle\right)_{i=1}^{m}=$ $\left(\int_{\Omega}\left\langle x_{i}^{\prime}, f(\omega)\right\rangle \mu(d \omega)\right)_{i=1}^{m}$ ou $f$ est une section Pettis-intégrable de $X$. Donc la convexité de $A$ découle du théorème de Ljapunov ([CV2, Theorem p. 118]).

Dans le théorème qui suit, on suppose que $E$ est un espace localement convexe séparé quasi-complet et qu'il existe une suite dans $E^{\prime}$ qui sépare les points de $E$.

Théorème 3.4. Soit $X: \Omega \rightarrow \operatorname{cwk}(E)$ une multifonction scalairement intégrable et soit $\left(f_{n}\right)_{n \in \mathbb{N}}$ une suite de sections Pettis-intégrable de $X$. On suppose que pour toute partie équicontinue $B \subset E^{\prime}$, l'ensemble $\left\{\delta^{*}\left(x^{\prime}, X\right): x^{\prime} \in B\right\}$ est relativement $\sigma\left(L^{1}, L^{\infty}\right)$ compact. Alors il existe une sous-suite $\left(f_{n_{k}}\right)_{k \in \mathbb{N}}$ et une application $f$ Pettis-intégrable telle que l'on ait pour tout $A \in \mathcal{F}$ et pour tout $x^{\prime} \in E^{\prime}$

$$
\left\langle x^{\prime}, \int_{A} f(\omega) \mu(d \omega)\right\rangle=\left\langle x^{\prime}, \lim _{m \rightarrow \infty} \int_{A} \frac{1}{m} \sum_{k=1}^{m}, f_{n_{k}}(\omega) \mu(d \omega)\right\rangle .
$$

De plus on a:

$$
f(\omega) \in \overline{\mathrm{co}} \mathrm{w}-\operatorname{Ls}\left(f_{n}(\omega)\right) \text { p.p. }
$$

Si on suppose de plus que $(\Omega, \mathcal{A}, \mu)$ est sans atomes et que $X$ est Pettis intégrable, alors pour tout voisinage $V$ de $\int_{\Omega} f(\omega) \mu(d \omega)$, il existe une application $f_{V}$, Pettis-intégrable, vérifiant $f_{V}(\omega) \in \mathrm{w}-\operatorname{Ls}\left(f_{n}(\omega)\right)$ p.p telle que $\int_{\Omega} f_{V}(\omega) \mu(d \omega) \in V$.

Démonstration: Soit $\left(e_{p}^{\prime}\right)_{p \in \mathbb{N}}$ une suite dense dans $E^{\prime}$ pour la topologie de Mackey ([CV2, Lemma III.32]). Comme pour tout $x^{\prime} \in E^{\prime}$ et pour tout $n \in \mathbb{N}$ on a

$$
\left|\left\langle x^{\prime}, f_{n}(\omega)\right\rangle\right| \leq\left|\delta^{*}\left(x^{\prime} ; X(\omega)\right)\right|+\left|\delta^{*}\left(x^{\prime} ;-X(\omega)\right)\right| .
$$

Pour tout $p$ fixé, la suite $\left(\left\langle e_{p}^{\prime}, f_{n}\right\rangle\right)_{n \in \mathbb{N}}$ est bornée dans $L_{\mathbb{R}}^{1}(\Omega, \mathcal{A}, \mu)$. Comme dans Balder [B2], par application du théorème de Komlos, il existe une application $\varphi_{p} \in L_{\mathbb{R}}^{1}(\Omega, \mathcal{A}, \mu)$, une sous-suite extraite de $\left(\left\langle e_{p}^{\prime}, f_{n}\right\rangle\right)_{n \in \mathbb{N}}$ que l'on notera $\left(\left\langle e_{p}^{\prime}, f_{n_{k}}\right\rangle\right)_{k \in \mathbb{N}}$ que:

$$
\lim _{k \rightarrow \infty} \frac{1}{k} \sum_{i=1}^{k}\left\langle e_{p}^{\prime}, f_{n_{k_{i}}}(\omega)\right\rangle=\varphi_{p}(\omega) \text { p.p pour tout } p
$$


où $\left(f_{n_{k_{i}}}\right)_{i \in \mathbb{N}}$ est une suite quelconque extraite de $\left(f_{n_{k}}\right)_{k \in \mathbb{N}}$ et l'ensemble négligeable dépend de la sous-suite $\left(f_{n_{k_{i}}}\right)_{i \in \mathbb{N}}$. Prenons pour $\left(f_{n_{k_{i}}}\right)_{i \in \mathbb{N}}$ la suite entière $\left(f_{n_{k}}\right)_{k \in \mathbb{N}}$ de ce qui précéde on obtient l'existence d'un négligeable $N$ tels que pour tout $p \in \mathbb{N}$ et pour tout $\omega \in \Omega \backslash N$, on ait:

$$
\lim _{m \rightarrow \infty} \frac{1}{m} \sum_{k=1}^{m}\left\langle e_{p}^{\prime}, f_{n_{k}}(\omega)\right\rangle=\varphi_{p}(\omega) .
$$

Soit $\omega \in \Omega \backslash N$ fixé. Comme $\frac{1}{m} \sum_{k=1}^{m} f_{n_{k}}(\omega) \in \Gamma(\omega)$ pour tout $m \in \mathbb{N}^{*}$ et que $\Gamma(\omega)$ est convexe faiblement compact, la suite $\left(\frac{1}{m} \sum_{k=1}^{m}\left\langle\cdot, f_{n_{k}}(\omega)\right\rangle\right)_{m \geq 1}$ est équicontinue pour la topologie de Mackey, il résulte donc du théorème d'Ascoli l'existence d'une application $f$ telle que pour tout $\omega \in \Omega \backslash N$ on ait:

$(* *) \quad \forall x^{\prime} \in E^{\prime}, \lim _{m \rightarrow \infty} \frac{1}{m} \sum_{k=1}^{m}\left\langle x^{\prime}, f_{n_{k}}(\omega)\right\rangle=\left\langle x^{\prime}, f(\omega)\right\rangle$.

Montrons que $f(\omega) \in \overline{\mathrm{co}} \mathrm{w}-\operatorname{Ls}\left(f_{n}(\omega)\right)$ p.p. Remarquons d'abord que si $(a)_{n \in \mathbb{N}}$ est une suite de réels telle que: $\lim _{n \rightarrow \infty} \frac{1}{n} \sum_{k=1}^{n} a_{i}=a$; alors pour tout $k \in \mathbb{N}$ on a $\lim _{n \rightarrow \infty} \frac{1}{n} \sum_{i=k}^{k+n} a_{i}=a$. En effet on a:

$$
\frac{1}{n} \sum_{i=k}^{k+n} a_{i}=\frac{n+k}{n}\left(\frac{1}{n+k} \sum_{i=1}^{k+n} a_{i}\right)-\frac{1}{n} \sum_{i=1}^{k-1} a_{i} .
$$

Le résultat s'obtient par passage à la limite sur $n$. D'où pour tout $x^{\prime} \in E^{\prime}$ et pour tout $k \in \mathbb{N}$; on a:

$$
\lim _{m \rightarrow \infty}\left\langle x^{\prime}, \frac{1}{m} \sum_{k=m}^{m+k} f_{n_{k}}(\omega)\right\rangle=\left\langle x^{\prime}, f(\omega)\right\rangle \text { p.p. }
$$

Par conséquent on a:

$$
\left\langle x^{\prime}, f(\omega)\right\rangle \in \cap_{k \in \mathbb{N}} \overline{\operatorname{CO}}\left\{\left\langle x^{\prime}, f_{n}(\omega)\right\rangle ; k \geq n\right\}=\operatorname{coLs}\left(\left\langle x^{\prime}, f_{n}(\omega)\right\rangle\right) .
$$

On déduit du Lemme 3.1 que:

$$
\left\langle x^{\prime}, f(\omega)\right\rangle \leq \delta^{*}\left(x^{\prime} ; \overline{\mathrm{co}} \mathrm{w}-\mathrm{Ls}\left(f_{n}(\omega)\right)\right. \text { p.p. }
$$


La relation étant vraie pour tout $x^{\prime} \in E^{\prime}$ et pour tout $\omega \in \Omega \backslash N$, on a:

$$
f(\omega) \in \overline{\mathrm{co}} \mathrm{w}-\operatorname{Ls}\left(f_{n}(\omega)\right) \text { p.p. }
$$

Reste à montrer que $f$ est Pettis-intégrable. Pour cela il suffit de montrer que $f$ vérifie les hypothèses du Théorème 3.2. L'hypothèse (2) découle de $(* *)$ et du théorème de Lebesgue-Vitali. La majoration

$$
\left|\left\langle x^{\prime}, f(\omega)\right\rangle\right| \leq\left|\delta^{*}\left(x^{\prime}, X(\omega)\right)\right|+\left|\delta^{*}\left(x^{\prime},-X(\omega)\right)\right|
$$

entraine que pour toute partie équicontinue $B \subset E^{\prime}$ l'ensemble $\left\{\left(\left\langle x^{\prime}, f\right\rangle\right) ; x^{\prime} \in B\right\}$ est relativement faiblement compact ([DS, Theorem IV 8.9]). Le point (1) est ainsi verifié.

Pour la deuxième partie de l'énoncé, remarquons d'abord que la multifonction $\mathrm{w}-\operatorname{Ls}\left(f_{n}(\omega)\right)$ est à valeurs non vides et que, en vertu des hypothèses toutes ses sections sont Pettis-intégrables. Donc il suffit de montrer que $\int_{\Omega} f(\omega) \mu(d \omega) \in \overline{\int_{\Omega} \mathrm{w}-\operatorname{Ls}\left(f_{n}(\omega)\right) \mu(d \omega)}$. Soit $x^{\prime} \in E^{\prime}$, on a grâce au théorème de Strassen ([CV2, Theorem V.14])

$$
\begin{aligned}
\left\langle x^{\prime}, \int_{\Omega} f(\omega) \mu(d \omega)\right\rangle & \leq \delta^{*}\left(x^{\prime}, \int_{\Omega} \overline{\mathrm{co}} \mathrm{w}-\operatorname{Ls}\left(f_{n}(\omega)\right) \mu(d \omega)\right) \\
& =\int_{\Omega} \delta^{*}\left(x^{\prime}, \overline{\mathrm{co}} \mathrm{w}-\operatorname{Ls}\left(f_{n}(\omega)\right) \mu(d \omega)\right) \\
& =\int_{\Omega} \delta^{*}\left(x^{\prime}, \mathrm{w}-\operatorname{Ls}\left(f_{n}(\omega)\right) \mu(d \omega)\right) \\
& =\delta^{*}\left(x^{\prime}, \overline{\int_{\Omega} \mathrm{w}-\operatorname{Ls}\left(f_{n}(\omega)\right) \mu(d \omega)}\right) .
\end{aligned}
$$

Comme d'après la Proposition 3.3, l'ensemble $\overline{\int_{\Omega} \mathrm{w}-\operatorname{Ls}\left(f_{n}(\omega)\right) \mu(d \omega)}$ est convexe l'assertion est démontrée.

Théorème 3.5. Soient $\left(X_{n}\right)_{n \in \mathbb{N}}$ et $Y$ des multifonctions à valeurs dans $\operatorname{cwk}(E)$ scalairement intégrables, on suppose que:

(1) Pour tout $n \in \mathbb{N} ; X_{n}$ est faiblement Pettis-intégrable.

(2) Pour tout $n \in \mathbb{N} ; X_{n}(\omega) \subset Y(\omega)$ p.p.

(3) Pour toute partie équicontinue $B \subset E^{\prime}$, l'ensemble $\left\{\delta^{*}\left(x^{\prime}, Y\right)\right.$ : $\left.x^{\prime} \in B\right\}$ est relativement $\sigma\left(L^{1}, L^{\infty}\right)$ compact.

On a alors

$$
\text { w - seq - Ls } \int_{\Omega} X_{n}(\omega) \mu(d \omega) \subset \int_{\Omega} \overline{\operatorname{co}} \mathrm{w}-\operatorname{Ls} X_{n}(\omega) \mu(d \omega) \text {. }
$$


Démonstration: Si $x \in \mathrm{w}$-seq-Ls $\int_{\Omega} X_{n}(\omega) \mu(d \omega)$, alors il existe une suite $\left(x_{n_{k}}\right)_{k \in \mathbb{N}}$ avec $x_{n_{k}} \in \int_{\Omega} X_{n_{k}}(\omega) \mu(d \omega)$ telle que pour tout $x^{\prime} \in E^{\prime}$, on ait $\left\langle x^{\prime}, x\right\rangle=\lim _{k \rightarrow+\infty}\left\langle x^{\prime}, x_{n_{k}}\right\rangle$. Par définition, on a pour tout $k, x_{n_{k}}=$ $\int_{\Omega} f_{n_{k}}(\omega) \mu(d \omega)$ ou $f_{n_{k}}$ est une section de $X_{n_{k}}$. Il est clair que la suite $\left(f_{n_{k}}\right)_{k \in \mathbb{N}}$ vérifie les hypothèses du Théorème 3.3 , par conséquent il existe une application $f$ Pettis-intégrable verifiant

$$
f(\omega) \in \overline{\mathrm{co}} \mathrm{w}-\operatorname{Ls}\left(f_{n}(\omega)\right) \subset \overline{\mathrm{co}} \mathrm{w}-\operatorname{Ls}\left(X_{n}(\omega)\right) .
$$

L'existence d'une suite dans $E^{\prime}$ d'une suite séparant les points de $E$ entraine que la topologie faible est métrisable sur les compacts faibles, il résulte de Hess ([H, Proposition 3.10]) que la multifonction $\overline{\mathrm{co}} \mathrm{w}-$ $\operatorname{Ls}\left(X_{n}(\cdot)\right)$ est scalairement mesurable et d'après ce qui précéde admet au moins une section Pettis-intégrable; donc $\int_{\Omega} \overline{\mathrm{co}} \mathrm{w}-\mathrm{Ls} X_{n}(\omega) \mu(d \omega)$ a un sens. Reste a montrer que $x=\int_{\Omega} f(\omega) \mu(d \omega)$. D'après le Théorème 3.3, on a:

$$
\begin{aligned}
\left\langle x^{\prime}, \int_{\Omega} f(\omega) \mu(d \omega)\right\rangle & =\lim _{m \rightarrow \infty}\left\langle x^{\prime}, \int_{\Omega} \frac{1}{m} \sum_{i=1}^{m}, f_{n_{k_{i}}}(\omega) \mu(d \omega)\right\rangle \\
& =\lim _{m \rightarrow \infty} \frac{1}{m} \sum_{i=1}^{m}\left\langle x^{\prime}, \int_{\Omega} f_{n_{k_{i}}}(\omega) \mu(d \omega)\right\rangle \\
& =\lim _{k \rightarrow \infty}\left\langle x^{\prime}, \int_{\Omega} f_{n_{k}}(\omega) \mu(d \omega)\right\rangle .
\end{aligned}
$$

Remarque. Si l'on suppose dans les énoncés précédent que la mesure $\mu$ est finie, en vertu du théorème de Dunford Pettis, l'hypothèse de relative faible compacité peut être remplacée par l'uniforme intégrabilité.

IV. Théorème de Lebesgue pour des multifonctions fortement Pettis-intégrable.

Dans ce paragraphe, $E$ est un espace de Banach séparable et toutes les multifonctions considérées sont à valeurs dans $\operatorname{ck}(E)$. L'espace $\operatorname{ck}(E)$ est classiquement muni de la distance de Hausdorff $h$ définie par:

$$
\begin{aligned}
h(A, B) & =\max \left(\sup _{x \in A} d(x, B) ; \sup _{x \in B} d(x, A)\right) \\
& =\sup _{x^{\prime} \in B_{E^{\prime}}}\left|\delta^{*}\left(x^{\prime} ; A\right)-\delta^{*}\left(x^{\prime} ; B\right)\right| .
\end{aligned}
$$


Lemme 4.1. Soit $X: \Omega \rightarrow \operatorname{ck}(E)$ une multifonction fortement Pettisintégrable, alors l'ensemble $C=\left\{\delta^{*}\left(x^{\prime} ; X\right): x^{\prime} \in B_{E^{\prime}}\right\}$ est $\sigma\left(L^{1}, L^{\infty}\right)$ compact.

Démonstration: Par hypothèse pour tout $A \in \mathcal{F}$, il existe un convexe compact $K(A)$ tel que pour tout $x^{\prime} \in E^{\prime}$, on a:

$$
\delta^{*}\left(x^{\prime} ; K(A)\right)=\int_{A} \delta^{*}\left(x^{\prime} ; X(\omega) \mu(d \omega)\right) .
$$

D'après Eberlein-Smulyan, il suffit de montrer que $C$ est séquentiellement faiblement compact. Soit $\left(\delta^{*}\left(x_{n}^{\prime} ; X\right)\right)_{n \in \mathbb{N}}$ une suite dans $C$. Comme $E$ est séparable; il existe une sous suite $\left(x_{n_{k}}^{\prime}\right)_{k \in \mathbb{N}}$ qui converge faiblement vers $x_{0}^{\prime}$. D'où en utilisant le fait que $X$ est fortement Pettis intégrable et le théorème de Banach-Dieudonné ([G2, p. 214]), on a pour tout $A \in \mathcal{F}$,

$$
\begin{aligned}
\lim _{k \rightarrow \infty} \int_{A} \delta^{*}\left(x_{n_{k}}^{\prime}, X(\omega) \mu(d \omega)\right) & =\lim _{k \rightarrow \infty} \delta^{*}\left(x_{n_{k}}^{\prime}, K(A)\right) \\
& =\delta^{*}\left(x_{0}^{\prime}, K(A)\right) \\
& =\int_{A} \delta^{*}\left(x_{0}^{\prime}, X(\omega) \mu(d \omega)\right)
\end{aligned}
$$

Le théorème ci-dessus est le résultat principal de ce paragraphe.

Théorème 4.2. Si $Y$ est une multifonction fortement Pettis intégrable, alors la multifonction $X$ définie par $X(\omega)=\overline{\mathrm{co}}[Y(\omega) \cup(-Y(\omega))]$ l'est aussi.

Démonstration: La multifonction $X$ est scalairement mesurable et scalairement intégrable car pour tout $\omega$ et pour tout $x^{\prime}$ on a

$$
\delta^{*}\left(x^{\prime}, X(\omega)\right)=\max \left(\delta^{*}\left(x^{\prime}, Y(\omega)\right) ; \delta^{*}\left(x^{\prime},-Y(\omega)\right)\right) .
$$

D'autre part, toute section scalairement intégrable de $X$ est Pettisintégrable. En effet pour toute $f \in S_{X}$, on a:

$$
\left|\left\langle x^{\prime}, f(\omega)\right\rangle\right| \leq \mid \delta^{*}\left(x^{\prime}, Y(\omega)|+| \delta^{*}\left(-x^{\prime}, Y(\omega) \mid\right. \text { p.p. }\right.
$$

Ce qui entraine en vertu du lemme précédent et de ([DS, Theorem IV 8.9]) que l'ensemble $\left\{\left\langle x^{\prime}, f\right\rangle: x^{\prime} \in B_{E^{\prime}}\right\}$ est relativement $\sigma\left(L^{1}, L^{\infty}\right)$ compact. Donc d'après le Théorème 3.1, $f$ est Pettis-intégrable. Reste à montrer que $X$ est fortement Pettis-intégrable. D'après la formule de Strassen ([CV2, Theorem V 14]), on a pour tout $A \in \mathcal{A}$, 
$\int_{A} \delta^{*}\left(x^{\prime}, X(\omega) \mu(d \omega)\right)=\delta^{*}\left(x^{\prime}, \int_{A} X(\omega \mu(d \omega))\right)$ et l'ensemble $\int_{A} X(\omega \mu(d \omega))$ est convexe faiblement compact. Pour terminer la preuve, montrons que cet ensemble est compact pour la topologie de la norme. Il suffit pour cela de montrer que l'application $x^{\prime} \rightarrow \int_{A} \delta^{*}\left(x^{\prime}, X(\omega) \mu(d \omega)\right)$ est continue sur $B_{E^{\prime}}$ pour la topologie faible $([\mathbf{C 1}$, Théorème 3$])$. Soit $\left(x_{n}^{\prime}\right)_{n \in \mathbb{N}}$ qui converge faiblement vers $x^{\prime}$. Grâce au théorème de Banach-Dieudonné on a: $\lim _{n \rightarrow \infty} \delta^{*}\left(x_{n}^{\prime}, X(\omega)\right)=\delta^{*}\left(x^{\prime}, X(\omega)\right)$ p.p. La majoration:

$$
\left|\delta^{*}\left(x^{\prime}, X(\omega)\right)\right| \leq\left|\delta^{*}\left(x^{\prime}, Y(\omega)\right)\right|+\left|\delta^{*}\left(-x^{\prime}, Y(\omega)\right)\right| \text { p.p. }
$$

Le Lemme 4.1 et ([DS, Theorem IV 8.9]) entrainent que la suite $\left(\delta^{*}\left(x_{n}^{\prime}, X(\cdot)\right)_{n \in \mathbb{N}}\right.$ est relativement $\sigma\left(L^{1}, L^{\infty}\right)$ compacte. D'où par Lebesgue-Vitali, on a:

$$
\begin{aligned}
\lim _{n \rightarrow \infty} \delta^{*}\left(x_{n}^{\prime} \int_{A} X(\omega) \mu(d \omega)\right) & =\lim _{n \rightarrow \infty} \int_{A} \delta^{*}\left(x_{n}^{\prime}, X(\omega)\right) \mu(d \omega) \\
& =\int_{A} \delta^{*}\left(x^{\prime}, X(\omega)\right) \mu(d \omega) \\
& =\delta^{*}\left(x^{\prime}, \int_{A} X(\omega) \mu(d \omega)\right)
\end{aligned}
$$

Comme conséquence du théorème précédent, on obtient le théorème de Lebesgue suivant:

Corolaire 4.3. Soient $\left(X_{n}\right)_{n \in \mathbb{N}}$ et $Y$ des multifonctions fortement Pettis intégrables et $X$ une multifonction scalairement intégrable, on suppose que:

(1) Pour tout $x^{\prime} \in E^{\prime}:\left|\delta^{*}\left(x^{\prime}, X_{n}(\omega)\right)\right| \leq\left|\delta^{*}\left(x^{\prime}, Y(\omega)\right)\right|$ p.p.

(2) $\lim _{n \rightarrow \infty} \delta^{*}\left(x^{\prime}, X_{n}(\omega)\right)=\delta^{*}\left(x^{\prime}, X(\omega)\right)$ p.p.

Alors $X$ est fortement Pettis-intégrable et pour tout $A \in \mathcal{A}, \int_{A} X_{n}(\omega) \mu(d \omega)$ converge pour la distance de Hausdorff vers $\int_{A} X(\omega \mu(d \omega))$.

Démonstration: Les hypothèses (1) et (2) entrainent que pour tout $x^{\prime} \in E^{\prime}:$

$$
\left|\delta^{*}\left(x^{\prime}, X(\omega)\right)\right| \leq\left|\delta^{*}\left(x^{\prime}, Y(\omega)\right)\right| \text { p.p. }
$$


Ceci implique que $X(\omega) \subset \overline{\mathrm{co}}[Y(\omega) \cup(-Y(\omega))]$ et donc que $X$ est fortement Pettis-intégrable (Théorème 4.2). Par Lebesgue-Vitali on a:

$$
\begin{aligned}
\lim _{n \rightarrow \infty} \delta^{*}\left(x^{\prime}, \int_{A} X_{n}(\omega \mu(d \omega))\right) & =\lim _{n \rightarrow \infty} \int_{A} \delta^{*}\left(x^{\prime}, X_{n}(\omega)\right) \mu(d \omega) \\
& =\int_{A} \delta^{*}\left(x^{\prime}, X(\omega)\right) \mu(d \omega) \\
& =\delta^{*}\left(x^{\prime}, \int_{A} X(\omega \mu(d \omega))\right)
\end{aligned}
$$

Les théorèmes de Banach-Dieudonné et d'Ascoli entrainent que la convergence a lieu aussi pour la distance de Hausdorff.

Remerciements. L'auteur tient à remercier le rapporteur pour ses nombreuses remarques qui ont permis d'améliorer substanciellement les résultats de ce papier.

\section{References}

[A1] A. Amrani, Entaillabilité et convergence dans l'espace $L^{1}$, J. Polish. Acad. Science 42 (1994), 229-236.

[AC] A. Amrani and C. Castaing, Weak compactness in Pettis integration, J. Polish. Acad. Science 44(2) (1997), 139-150.

[ACV1] A. Amrani, C. Castaing and M. Valadier, Méthodes de troncatures apliquées à des problèmes de convergence faible où forte dans $L^{1}$, Arch. Rational Mech. Anal. 117 (1991), 147-156.

[ACV2] A. Amrani, C. Castaing and M. Valadier, Convergence forte impliquée par la convergence faible. Méthodes de troncature, C. R. Acad. Sci. Paris Sér. I Math. 314 (1992), 91-94.

[Ar] Z. Artstein, A note on Fatou's lemma in several dimensions, J. Math. Econom. 6 (1979), 277-282.

[Au] R. J. Aumann, Integral of set valued functions, J. Math. Anal. Appl. 12 (1965), 1-12.

[B1] E. J. BALDER, Fatou's lemma in infinite dimensional spaces, J. Math. Anal. Appl. 136 (1988), 450-465.

[B2] E. J. BALDER, New sequential compactness results for spaces of scalarly integrable functions, J. Math. Anal. Appl. 151 (1990), $1-16$. 
[BH] E. J. BALDER AND C. Hess, Fatou's lemma for multifunctions with unbounded values, Math. Oper. Res. 20(1) (1995), 175-188.

[C1] C. Castaing, Quelques applications du théorème de BanachDieudonné à l'intégration, Preprint.

[C2] C. Castaing, Quelques résultats de convergence des suites adaptées, Sém. d'Anal. convexe, Montpellier, Exposé 2 (1987).

[CC] C. Castaing and P. Clauzure, Lemme de Fatou multivoque, Atti Sem. Mat. Fis. Univ. Modena XXXIX (1991), 303-320.

[Co] A. Costé, Contribution à la théorie de l'intégration multivoque, Thése d'état, Paris (1977).

[CV1] C. Castaing and M. Valadier, Equations differentielles multivoques dans les espaces vectoriels localement convexes, R.I.R.O. 16 (1969), 3-16.

[CV2] C. Castaing And M. Valadier, "Convex analysis and measurable multifunctions," Lecture Notes in Math. 580, SpringerVerlag, Berlin, 1977.

[DS] N. Dunford And J. T. Schwartz, "Linear operators," Part I, Interscience, New York, 1964.

[G1] A. Grothendieck, Critères de compacité généraux dans les espaces fonctionnels, Amer. J. Math. 74 (1952), 168-186.

[G2] A. Grothendieck, Espaces vectoriels topologiques, Sao Paulo.

[Ge] R. Geitz, Pettis-integration, Proc. Amer. Math. Soc. 82 (1981), 81-86.

[H] C. Hess, Measurability and integrability for the upper limit of a sequence of multifonctions, J. Math. Anal. Appl. 153 (1990), 226-249.

[Hu] R. Huff, Remarks on Pettis integration, Proc. Amer. Math. Soc. 96 (1986), 402-404.

[K] G. Кнӧтне, “Topological vector spaces,” I, Springer-Verlag, 1969.

[Ko] J. Komlos, A generalization of a problem of Steinhaus, Acta Math. Hungar. 18 (1967), 217-229.

[M] K. Musial, Vitali and Lebesgue theorems for Pettis integral in locally convex spaces, Atti Sem. Mat. Fis. Univ. Modena 25 (1987), $159-166$. 
[Y] N. C. YAnnelis, Weak sequential convergence in $L^{p}(\mu, X)$, J. Math. Anal. Appl. 141 (1989), 72-83.

\author{
Departement de Mathématiques \\ Case 051 \\ Universite 2 \\ Montpellier \\ FRANCE
}

Primera versió rebuda el 15 d'abril de 1996 ,

darrera versió rebuda el 23 d'octubre de 1997 